\title{
Activation of neutral sphingomyelinase 2 by starvation induces cell-protective autophagy via an increase in Golgi- localized ceramide
}

\author{
Moon Jung Back', Hae Chan Ha', Zhicheng Fu', Jong Min Choi ${ }^{1,2}$, Yongwei Piao ${ }^{1}$, Jong Hoon Won ${ }^{1,3}$, Ji Min Jang ${ }^{1}$, \\ In Chul Shin ${ }^{1}$ and Dae Kyong Kim
}

\begin{abstract}
Autophagy is essential for optimal cell function and survival, and the entire process accompanies membrane dynamics. Ceramides are produced by different enzymes at different cellular membrane sites and mediate differential signaling. However, it remains unclear which ceramide-producing pathways/enzymes participate in autophagy regulation under physiological conditions such as nutrient starvation, and what the underlying mechanisms are. In this study, we demonstrate that among ceramide-producing enzymes, neutral sphingomyelinase 2 (nSMase2) plays a key role in autophagy during nutrient starvation. nSMase 2 was rapidly and stably activated upon starvation, and the enzymatic reaction in the Golgi apparatus facilitated autophagy through the activation of p38 MAPK and inhibition of mTOR. Moreover, nSMase2 played a protective role against cellular damage depending on autophagy. These findings suggest that nSMase2 is a novel regulator of autophagy and provide evidence that Golgi-localized ceramides participate in cytoprotective autophagy against starvation.
\end{abstract}

\section{Introduction}

Autophagy is a highly conserved intracellular catabolic process that degrades cytoplasmic components within lysosomes ${ }^{1}$. Autophagy protects cells under nutrientdeprived conditions by recycling cytoplasmic materials, and removes excess or toxic cellular components ${ }^{2}$. Dysfunction of the homeostatic role of autophagy contributes to the progression of various diseases, including neurodegenerative diseases ${ }^{3}$. The accumulation of toxic protein aggregates is considered the main cause of Parkinson's disease (PD) and Alzheimer's disease $(\mathrm{AD})^{4}$. Thus,

Correspondence: Dae Kyong. Kim (kimdk@cau.ac.kr)

${ }^{1}$ Department of Environmental and Health Chemistry, College of Pharmacy, Chung-Ang University, 84 Heukseok-ro, Dongjak-gu, Seoul 06974, Republic of Korea. ${ }^{2}$ Present address: Department of Molecular and Cellular Biology, Baylor College of Medicine, Houston, TX 77030, USA

Edited by: E. Baehrecke autophagy-regulating molecules are attractive therapeutic targets for neurodegenerative diseases.

Autophagy processes accompany membrane dynamics, starting with the initial step of an isolated cup-shaped, double-membrane structure termed the phagophore in the cytoplasm. This phagophore expands in size and eventually engulfs cytoplasmic cargo to form an autophagosome ${ }^{1}$. The autophagosome delivers the cargo to the lysosome by fusing to form an autolysosome, after which the cargo is degraded. During autophagy, the membrane undergoes continuous remodeling including curvature formation, budding, fission, and fusion ${ }^{5}$. The entire autophagy process relies on the regulation of membrane dynamics.

Ceramides are the central molecules of sphingolipid metabolism. They are constituents of cellular organelle membranes that mediate membrane dynamics by altering membrane fluidity or rigidity and also function as 


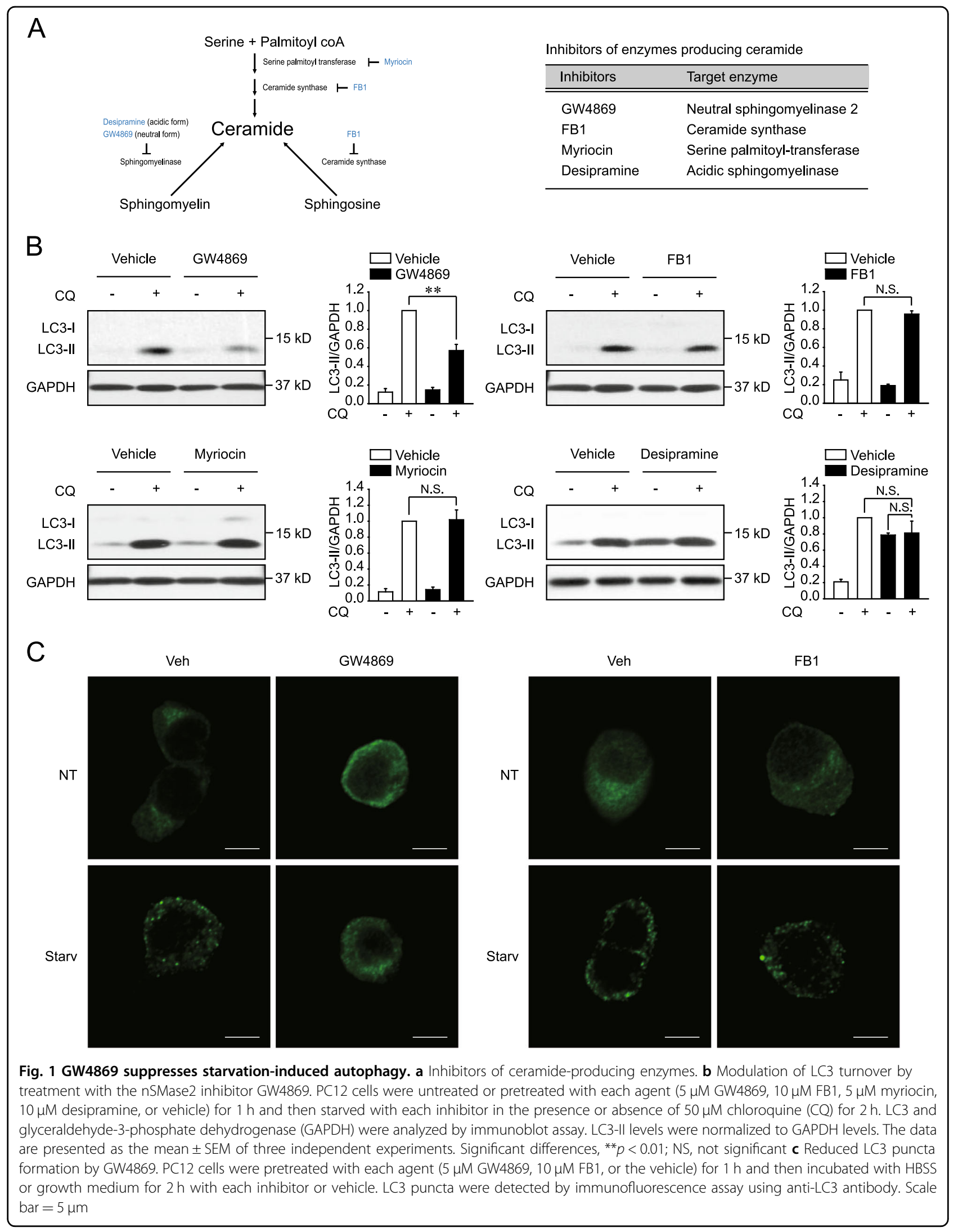


signaling molecules ${ }^{6}$. Ceramides are generated via various enzyme-mediated pathways in different cellular organelles. Ceramides can be synthesized by the condensation of serine and palmitoyl-CoA, catalyzed by serine palmitoyl transferase (SPT) and ceramide synthase (CerS; de novo pathway) in the endoplasmic reticulum $(E R)^{7}$. Another ceramide-producing pathway is the sphingomyelin (SM) pathway, in which SM is hydrolyzed to ceramides by neutral sphingomyelinase 2 (nSMase2) in the Golgi complex and plasma membrane ${ }^{8,9}$ or by acidic sphingomyelinase (aSMase) in the lysosome ${ }^{10}$. The ceramide production pathway determines the diverse physiological roles of ceramides in cellular processes, including cell proliferation, differentiation, and apoptosis ${ }^{11-15}$. Ceramides have been proposed to act as regulators of autophagy ${ }^{16-18}$. However, which ceramide-producing pathway participates in autophagy under physiological conditions, such as starvation, remain unclear.

nSMase2 is a $\mathrm{Mg}^{2+}$ - and phosphatidylserine-dependent neutral form of SMase. It is the major isoform of nSMase $^{19}$ and is highly expressed in the brain and bone ${ }^{20}$. nSMase2 has been studied concerning cell growth and development ${ }^{20-22}$, and plays pivotal role in exosome release via the control of exosome budding into multivesicular endosomes ${ }^{23,24}$. However, the role of nSMase2 in autophagy remains unknown.

In this study, we identified nSMase 2 as a key enzyme that mediates starvation-induced autophagic flux, among other ceramide-producing enzymes, through its stable activation and increase in Golgi-localized ceramides. nSMase 2 protected cells against various toxic stimuli, including starvation and dopaminergic toxicity. Additionally, the expression of nSMase 2 in the brain of old mice with reduced autophagy was decreased compared with that in young mice. In GEO database analysis, the expression of nSMase2 in the substantia nigra was significantly lower in patients with PD than in healthy donors, and was correlated with autophagy-related genes (ATGs). Overall, these findings demonstrate that nSMase2 mediates autophagy and has a cytoprotective role, providing essential insights into the complexity of the mechanisms underlying autophagy regulation. This further suggests that nSMase2 may serve as a novel therapeutic target for treating autophagy-associated diseases, especially PD.

\section{Results}

\section{nSMase2 mediates starvation-induced autophagy}

To investigate the ceramide-producing enzyme or pathway involved in the regulation of autophagy, autophagic flux was analyzed in the presence of various inhibitors of ceramide-generating enzymes (Fig. 1a) in rat neuroblastoma PC12 cells, which feature a well-conserved sphingolipid metabolism (Supplementary Figure 1A).
Autophagic flux was induced by starvation in Hank's balanced salt solution (HBSS) and was measured by determining the turnover of LC3. The assay compares the levels of the autophagosome marker, LC3-II, in the presence or absence of the lysosomal inhibitor, chloroquine (CQ), to estimate the degree of autophagic LC3 degradation $^{25}$. Starvation-induced LC3 turnover was suppressed by the nSMase2 inhibitor GW4869 (Fig. 1b). Furthermore, starvation-induced LC3 puncta formation, which is associated with autophagosome or autolysosome formation, was diminished by treatment with GW4869 (Fig. 1c). However, the de novo pathway inhibitors, CerS inhibitor (fumonisin B1) and SPT inhibitor (myriocin), had negligible effect on starvation-induced LC3 turnover (Fig. 1b). Treatment with the aSMase inhibitor desipramine increased LC3-II levels in the absence of CQ, while LC3-II levels in the presence of CQ were similar to those of the vehicle-treated control group (Fig. 1b). The data indicate that the inhibitor acted as a lysosomal inhibitor. Thus, aSMase may be required for lysosomal function rather than for autophagic flux, which is consistent with results from previous studies ${ }^{26,27}$.

nSMase2 has been extensively studied among the neutral forms of SMase because of its actual and major physiological activity $^{28}$. To examine the role of nSMase2 in starvation-induced autophagy, gene modulation experiments were conducted. Knockdown or overexpression of nSMase2 was confirmed by immunoblot analysis (Supplementary Figure 1B). Similar to nutrient starvation, overexpression of V5-tagged nSMase2 induced LC3 puncta formation, LC3 turnover, and degradation of the autophagy substrate p62 in V5-positive cells (Fig. 2a). Furthermore, the knockdown of Smpd3, which encodes nSMase2, by a pool of two small interfering RNAs (siRNAs) suppressed starvation-induced LC3 puncta formation, p62 degradation, and LC3 turnover (Fig. 2b). The inhibitory effect of nSMase 2 knockdown on starvationinduced autophagic flux was also confirmed using the individual Smpd3 siRNA and the additional pool of four other siRNAs (Supplementary Figure 2); inhibition was not due to off-target effects. The data imply that nSMase2 mediates starvation-induced autophagy.

GW4869 inhibits the enzyme activity of nSMase $2^{29}$ and suppresses autophagy (Fig. 1), implying the requirement of nSMase 2 activity for autophagy. To examine if the autophagy-inducing effects of nSMase2 involve its enzymatic activity, levels of autophagic flux were compared between cells expressing two catalytically inactive mutants (D428A, impaired substrate recognition; H639A, null in hydrolytic activity) and wild-type (WT) cells ${ }^{8}$. WT V5-tagged nSMase2 transfection induced autophagic flux as indicated by LC3 puncta formation, LC3 turnover, and p62 degradation. The induced flux was absent in both inactive mutants (Fig. 2c). nSMase2 overexpression in 


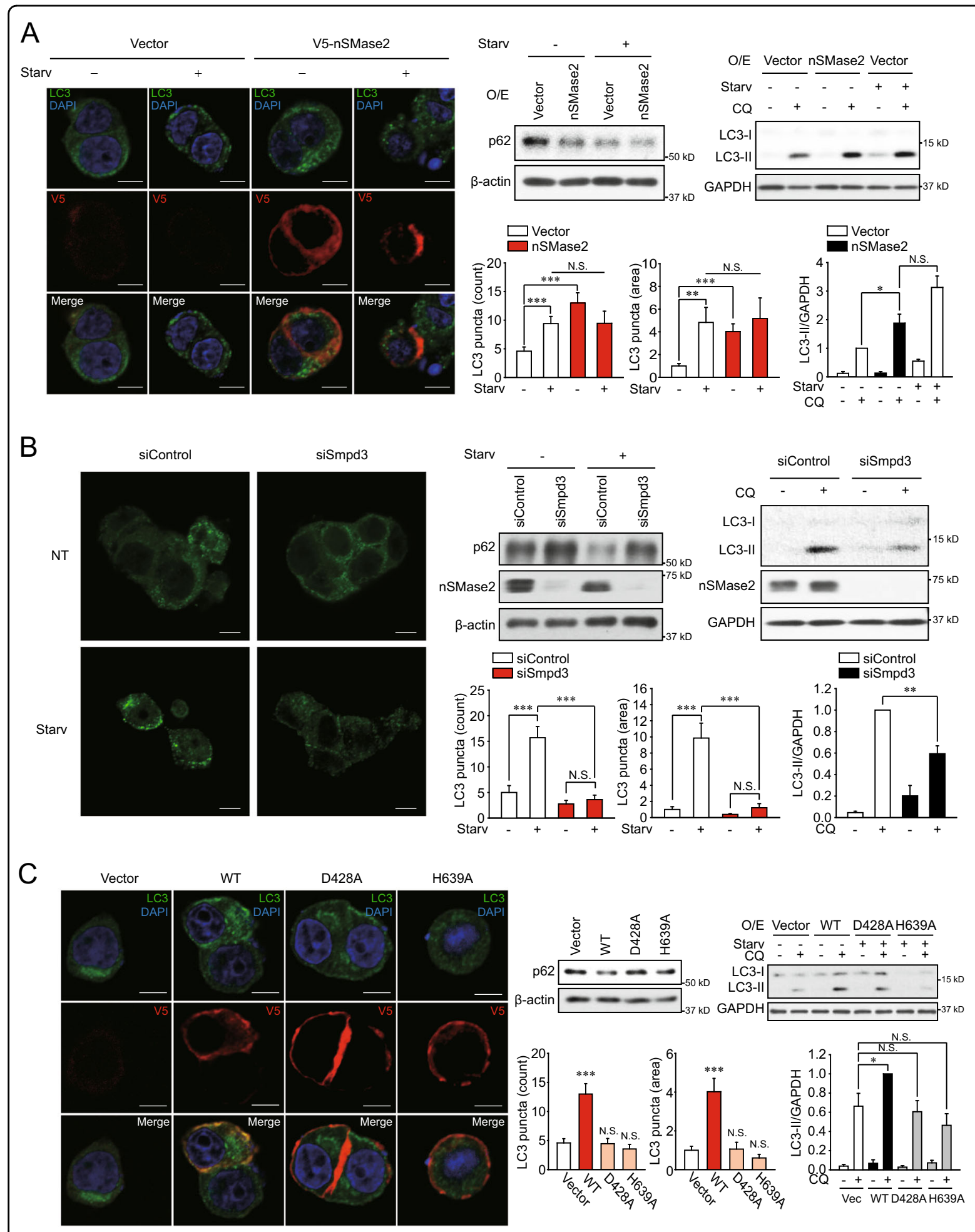

Fig. 2 (See legend on next page.) 
(see figure on previous page)

Fig. 2 nSMase2 induces autophagy. a Enhanced autophagic flux by overexpression of nSMase2. PC12 cells were transfected with $1 \mu \mathrm{g}$ plasmidencoding V5-tagged nSMase2 or empty vector. b Reduced autophagic flux by knockdown of nSMase2. PC12 cells were transfected with $50 \mathrm{nM}$ nSMase2 siRNA (siSmpd3) or non-targeting negative control siRNA (siControl). c Catalytically inactive mutants of nSMase2 were unable to induce autophagy. PC12 cells were transfected with either plasmid-encoding V5-tagged WT nSMase2, catalytically inactive forms of nSMase2 (D428A and H639A), or empty vector. At $48 \mathrm{~h}$ after transfection, the cells were incubated with HBSS or growth medium for $2 \mathrm{~h}$. Autophagic flux was determined by the levels of LC3 puncta formation and p62 degradation. For the LC3 puncta counting assay of the overexpression group, LC3 puncta were counted in only V5-positive cells. For the LC3 turnover assay, transfected cells were starved with HBSS with or without $50 \mu \mathrm{M}$ CQ for $2 \mathrm{~h}$, and LC3 and GAPDH were analyzed by immunoblotting. LC3-II levels were normalized to GAPDH levels, and the quantified immunoblot assay data are presented as the mean \pm SEM of at least three independent experiments. Scale bar $=5 \mu \mathrm{m}$; Significant differences between the indicated groups, ${ }^{*} p<0.05,{ }^{* *} p$ $<0.01$ and ${ }^{* *} p<0.001$; NS, not significant

H639A, but not D428A, tended to decrease LC3 turnover compared to the vector transfected control, although the difference was not statistically significant. The finding was likely a dominant-negative effect of the H639A mutant, because the D428A mutant is defective in substrate binding, while H639A mutant that is impaired in hydrolytic activity could compete with endogenous nSMase 2 for the binding of substrates. The absence of nSMase2 activity in the inactive mutants was confirmed by transiently overexpression (Supplementary Figure 3). These results suggest that nSMase 2 may induce autophagy through its enzymatic activity.

\section{Starvation activates nSMase2 and increases ceramide formation in the Golgi apparatus}

Considering the positive role of nSMase 2 in autophagy induction, we next examined the activity or expression of nSMase2 during nutrient starvation. Both specific activity and protein expression of nSMase 2 significantly increased under starvation, accompanied by autophagy, within 15-30 min after starvation (Fig. 3a-c). The rapid changes in nSMase2 expression suggested that regulation might occur at the post-transcriptional level, rather than the transcriptional level. As expected, mRNA levels of Smpd3 were not altered during starvation (Fig. 3d). A previous study described enhanced protein stability and activity of nSMase2 by phosphorylation at multiple serine sites, which was regulated by protein phosphatase $2 \mathrm{~B}(\mathrm{PP} 2 \mathrm{~B})^{30}$. Presently, the serine phosphorylation level of nSMase 2 in starved cells was assessed by the nSMase 2 pull-down assay. As expected, serine phosphorylation of nSMase2 increased during starvation (Fig. 3e).

In cells, nSMase 2 is mainly localized to the Golgi apparatus and plasma membrane ${ }^{8,9}$, while CerS is localized to the $\mathrm{ER}^{7}$. Thus, localization of ceramides in nutrient-starved cells was assessed by immunofluorescence (IF). During nutrient starvation with HBSS, increased co-localization of ceramides and giantin (the Golgi marker) was observed (Fig. 4a). However, there were no observable changes in ER-localized ceramide levels (Fig. 4b). The co-localization of ceramides and Golgi or ER marker were quantified using Pearson's correlation coefficient, with 1 and 0 indicating perfect and no correlation, respectively (Fig. 4c). In addition, colocalization of ceramides and nSMase 2 was induced by starvation (Fig. 4d). The specificity of antibody binding to nSMase2 during IF assays was confirmed in V5-tagged nSMase2-overexpressing cells (Supplementary Figure 4).

\section{Inhibition of ceramide transfer protein (CERT) or sphingomyelin synthase (SMS) represses starvation- induced autophagic flux}

To evaluate if localization of ceramides to the Golgi complex is required to trigger autophagy, we examined the effects of a CERT inhibitor (HPA-12) and an SMS inhibitor (D609) on starvation-induced autophagic flux. As depicted in Fig. 5a, CERT transfers the de novosynthesized ceramide from the ER to the Golgi complex ${ }^{31}$, followed by the synthesis of SM by SMS1 using the transferred ceramide ${ }^{32}$. HPA-12 and D609 limited the formation of SM by retaining ceramides in the ER, preventing their use by nSMase $2^{33,34}$. As expected, treatment with HPA-12 or D609 significantly reduced the starvation-induced autophagic flux (Fig. 5b).

We also checked whether the ceramide supply could rescue the suppressed autophagic flux by inhibition of ceramide transport from the ER to the Golgi complex. Treatment of the C2- and C6-ceramides that reach Golgi complex $^{35}$ significantly increased the autophagic flux inhibited by HPA-12 (Fig. 5c). The data imply that the Golgi-localized ceramide is required for induction of autophagy. Collectively, these results support the notion that production of ceramides by nSMase 2 in the Golgi apparatus plays a key role in autophagy.

\section{nSMase2-mediated induction of autophagy occurs via activation of p38 MAPK and inhibition of mTOR}

To explore the downstream pathways of nSMase 2 involved in autophagy regulation, we examined the effects of nSMase 2 on phosphorylation of p38 mitogen-activated protein kinase (MAPK), mechanistic target of rapamycin (mTOR), Akt, c-Jun N-terminal kinase (JNK) 1/2, AMPactivated protein kinase (AMPK), and Unc-51 like autophagy activating kinase (ULK1). All are signaling 
A

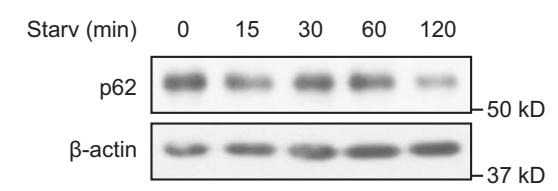

Starv $(\min ) \quad 0 \quad 15 \quad 30 \quad 60 \quad 120$
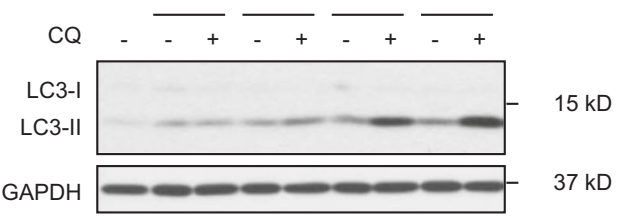

C

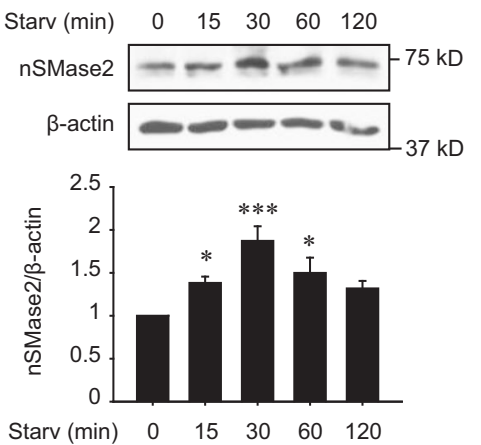

B

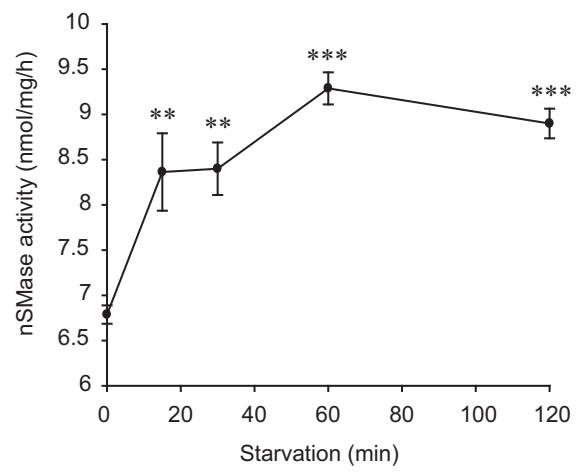

$\mathrm{E}$
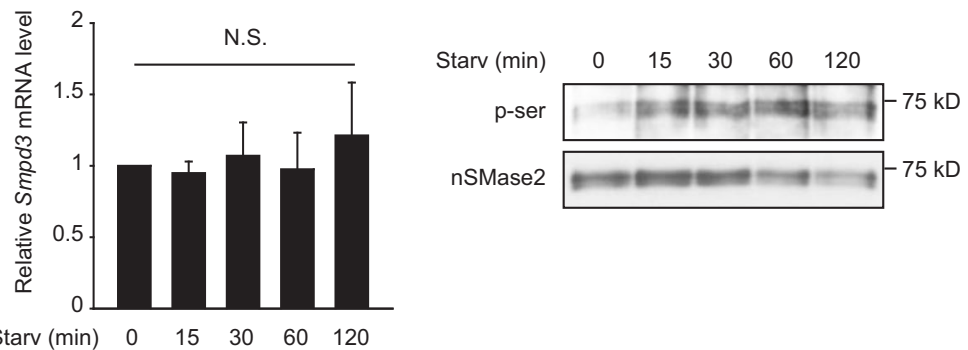

Fig. 3 Nutrient starvation upregulates nSMase2. PC12 cells were starved with HBSS for the indicated times. a Induction of autophagy by nutrient starvation in PC12 cells. Degradation of p62 and LC3 turnover were detected by immunoblotting for assessing autophagic flux. For LC3 turnover assays, cells were starved with HBSS with or without $50 \mu \mathrm{M} C Q$ for the indicated times. b Activation of nSMase2 by starvation. Specific activity of nSMase2 was analyzed using $\left[{ }^{14} \mathrm{C}\right]$-labeled sphingomyelin. $\mathbf{c}$ Increase in nSMase2 expression by starvation. Protein expression levels of nSMase2 in starved cells were determined by immunoblotting and were normalized to $\beta$-actin levels. $\mathbf{d}$ No changes in nSMase2 mRNA levels were induced by starvation. The mRNA levels of nSMase2 were measured by quantitative real-time PCR and were normalized to Hprt1. e Starvation-induced phosphorylation of nSMase2 at a serine residue. PC12 cells were starved with HBSS for the indicated time, and cell lysates were incubated with biotinconjugated sphingomyelin (the nSMase2 substrate) followed by pull-down with streptavidin-sepharose beads. The pellets were analyzed using immunoblots to detect serine phosphorylation of nSMase2. The data are presented as the mean \pm SEM of three independent experiments. Significant differences, ${ }^{*} p<0.05,{ }^{* *} p<0.01$, and ${ }^{* * *} p<0.001$ (one-way ANOVA followed by LSD test); NS, not significant

molecules reported to regulate the autophagy machinery. The Akt-mTOR pathway is a crucial negative regulator of autophagy $^{36}$, while the JNK-beclin 1 and the AMPKULK1 pathways are positive regulators ${ }^{37,38}$. Although the role of p38 MAPK in autophagy is still controversial, one study reported starvation-induced autophagy mediated by p38 MAPK $^{39}$. Phosphorylation of p38 MAPK increased during starvation in HBSS, and starvation-induced p38 MAPK phosphorylation was reduced by nSMase2 knockdown (Fig. 6a). Akt is known as an upstream activator of $\mathrm{mTOR}^{40}$, and ceramides are regarded as mTOR regulators via Akt inhibition ${ }^{41}$. However, starvationinduced reduction of mTOR phosphorylation was suppressed by siRNA knockdown of nSMase2, without a decrease in Akt phosphorylation (Fig. 6a). The phosphorylation level of Akt was unaltered, even by nSMase2 overexpression (Supplementary Figure 5A). JNK1/2 is also modulated by ceramide treatment ${ }^{16}$, but starvation- induced JNK1/2 phosphorylation was not suppressed by transfection with nSMase2 siRNA (Fig. 6a). AMPK direct activates ULK1 by phosphorylating Ser317, which promotes autophagy $^{38}$, and phosphorylation of AMPK is affected by ceramide treatment ${ }^{42-44}$. However, the enhanced phorphorylation levels of AMPK and ULK1 (Ser 317) by starvation were not reduced by nSMase 2 knockdown, while the transfection with nSMase2 siRNA rather increased phosphorylation of ULK1 at Ser 317 in the basal nutrition condition (Fig. 6a). Additionally, overexpression of nSMase2 enhanced p38 MAPK phosphorylation and reduced mTOR phosphorylation (Fig. 6b). Notably, the effects of wild-type nSMase2 overexpression on p38 MAPK and mTOR phosphorylation were not detected for the enzymatically inactive mutants of nSMase2 (Fig. 6b). The data indicate that nSMase2 activates p38 MAPK and inhibits mTOR signaling. p38 MAPK inhibition by SB203580 suppressed the LC3 puncta formation triggered by nSMase 2 overexpression 
A

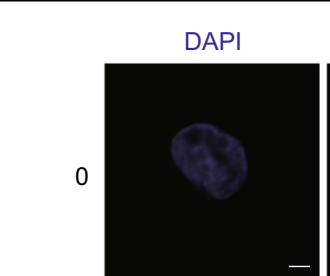

Giantin

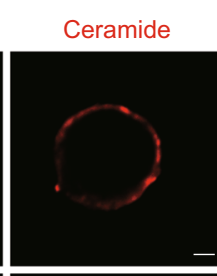

Merge
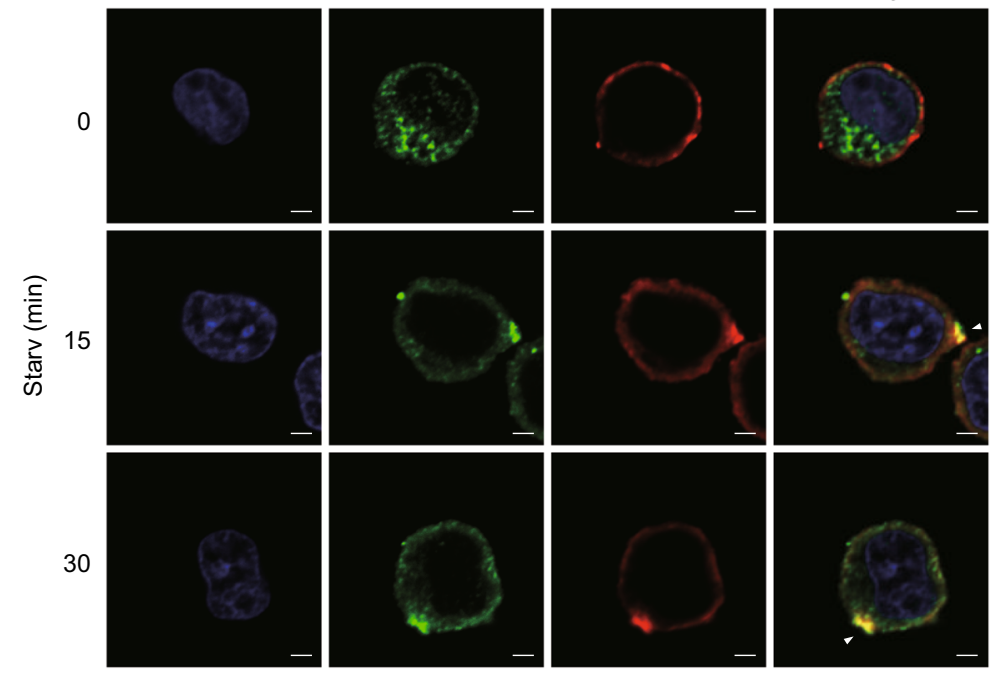

B

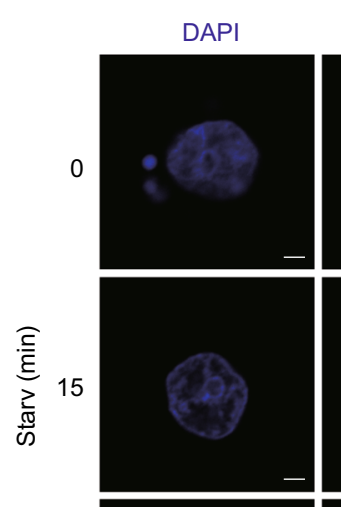

\section{Calnexin}

Ceramide
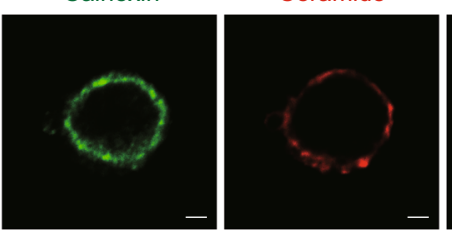

Merge
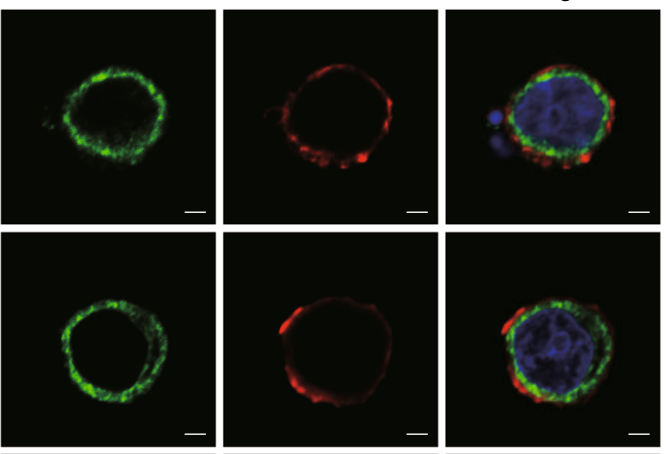

30
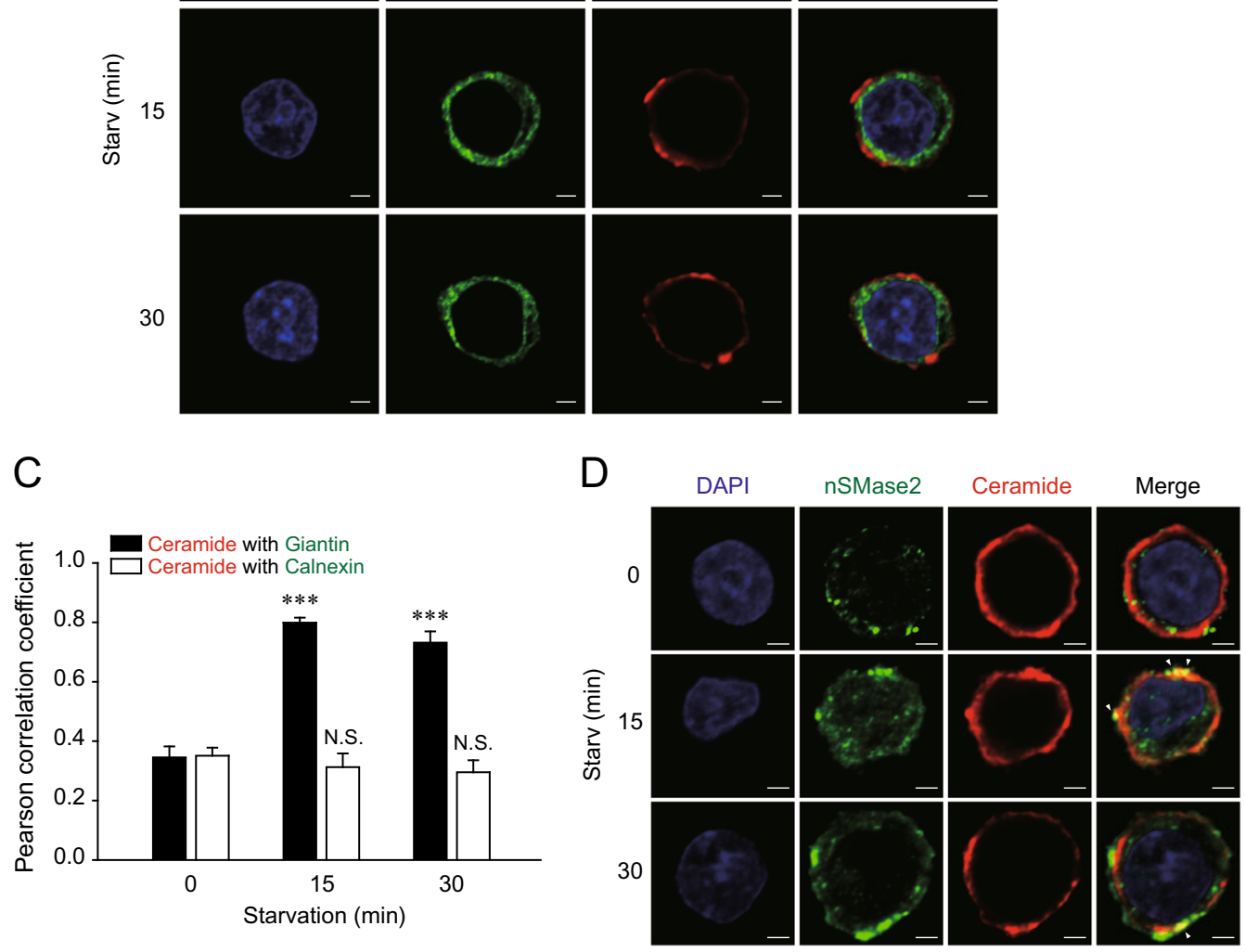

Fig. 4 Nutrient starvation increases ceramide formation in the Golgi apparatus. PC12 cells were starved with HBSS for the indicated times. a Golgi-localized ceramides were observed during starvation. b No observable changes in ER-localized ceramide levels were evident during starvation. c The co-localization levels between ceramide and Golgi or ER marker were quantified using ImageJ software for more than 10 cells per each group. Significant differences, ${ }^{* * *} p<0.001$; NS, not significant $\mathbf{d}$ Increased co-localization of ceramide with nSMase2 is triggered by starvation. Starved cells were co-stained with antibodies against ceramide and the Golgi marker giantin, ER marker calnexin, or nSMase2. Yellow regions indicated by arrows in the merged images represent co-localization of green-stained nSMase2 or each organelle marker and red-stained ceramide. Scale bar $=2 \mu \mathrm{m}$ 
A

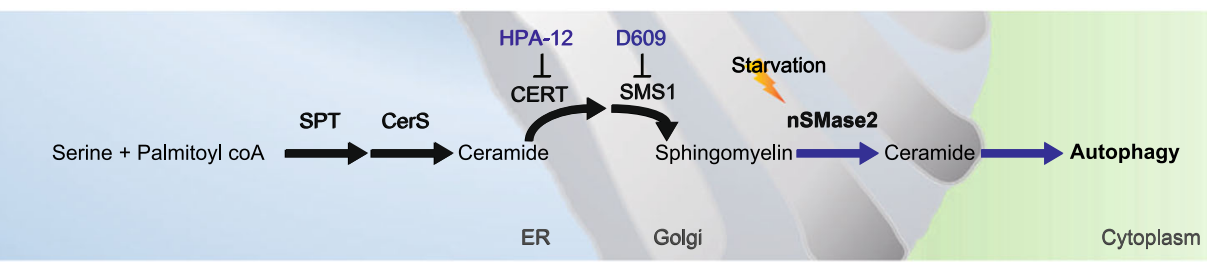

$\mathrm{B}$
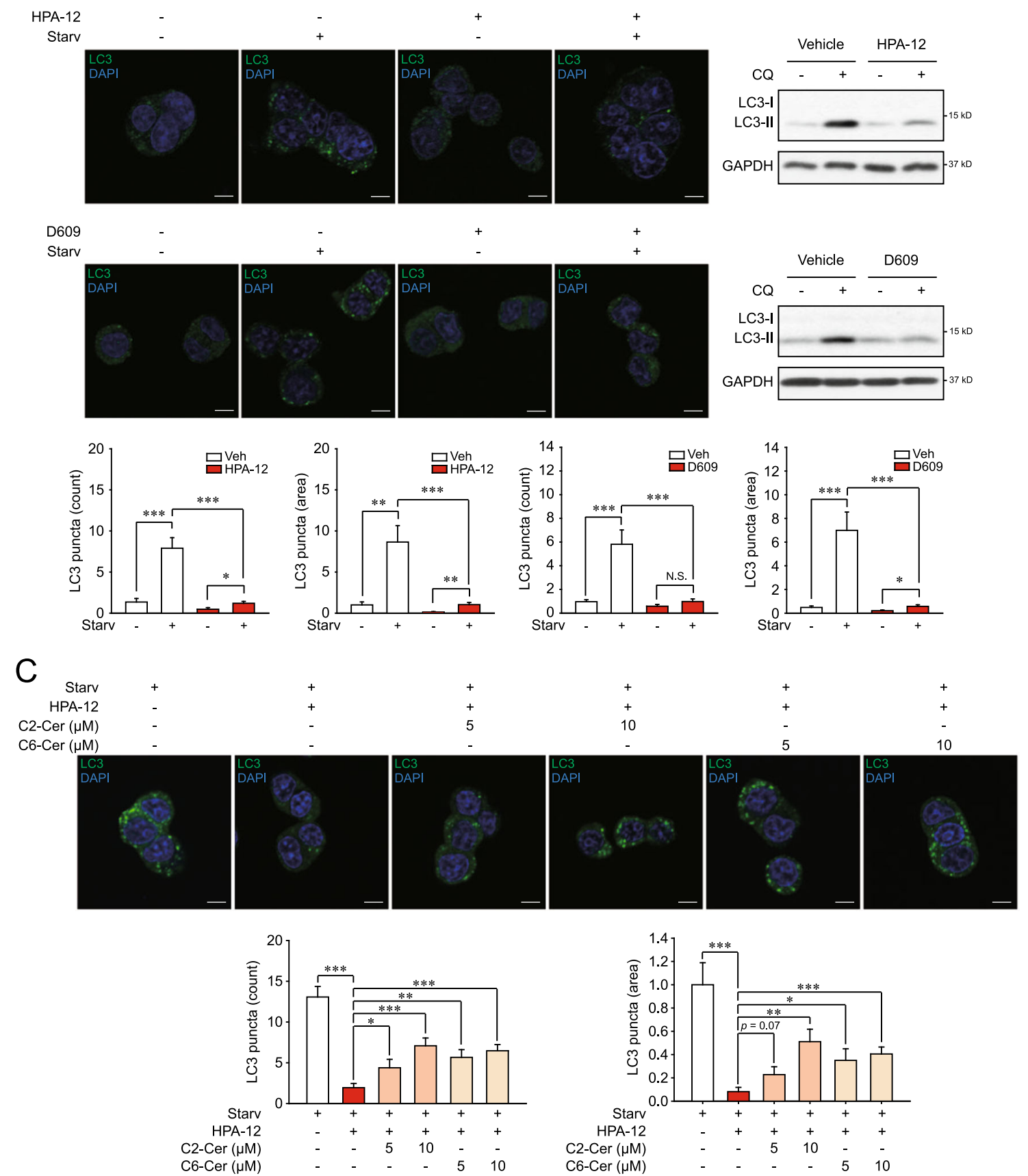

Fig. 5 (See legend on next page.) 
(see figure on previous page)

Fig. 5 HPA-12 and D609 suppresses starvation-induced autophagy. a A schematic diagram illustrating the proposed model in which the enzymatic reaction by nSMase2 is a key step in regulating autophagy. $\mathbf{b}$ Decreased autophagic flux by either CERT or SMS inhibition. PC12 cells were pretreated with $10 \mu \mathrm{M} \mathrm{HPA}-12,100 \mu \mathrm{M}$ D609 or the vehicle for $1 \mathrm{~h}$ and then starved for $2 \mathrm{~h}$ in the presence or absence of $50 \mu \mathrm{M} \mathrm{CQ}$ and $10 \mu \mathrm{M} \mathrm{HPA}$ or $100 \mu \mathrm{M}$ D609. LC3 puncta formation and LC3 turnover were analyzed to determine autophagic flux. c Recovery of CERT inhibition-attenuated autophagic flux by ceramide supply. PC12 cells were pretreated with $10 \mu \mathrm{M} \mathrm{HPA}-12$ or the vehicle for $1 \mathrm{~h}$ and then starved for $2 \mathrm{~h}$ with $\mathrm{C} 2$-ceramide or C6-ceramide at the indicated concentrations in the presence or absence of $10 \mu \mathrm{M} \mathrm{HPA}-12$. Autophagic flux was measured by LC3 puncta formation. Scale bar $=5 \mu \mathrm{m}$; Significant differences between the indicated groups, ${ }^{*} p<0.05,{ }^{* *} p<0.01$, and ${ }^{* * *} p<0.001$; NS, not significant

(Fig. 6c). Furthermore, the nSMase2-induced p62 degradation was repressed by inhibition of p38 MAPK (Supplementary Figure 5C). Rapamycin-mediated inhibition of mTOR partially reversed the effect of nSMase 2 knockdown on LC3 puncta formation during starvation (Fig. 6d), although this reversal effect of rapamycin was not observed in the p62 degradation assay (Supplementary Figure 5D). The inhibitory effects of SB203580 and rapamycin on p38 MAPK and mTOR, respectively, were valid at the concentrations used in the experiments (Supplementary Figure 5B). Together, these results indicate that nSMase2-mediated induction of autophagy occurs through signaling pathways via p38 MAPK activation and mTOR inhibition.

\section{nSMase2 plays a protective role against toxic stress} depending on autophagy and its levels are reduced in PD

Since autophagy primarily acts as a survival mechanism against various stressors, we examined if $\mathrm{nSMase} 2$ has a protective function in stress-induced cytotoxicity through induction of autophagy. Starvation-induced cell death, measured by lactate dehydrogenase (LDH) release, increased after nSMase 2 knockdown (Fig. 7a). Moreover, the number of propidium iodide (PI)-positive (dead) cells also increased during starvation after nSMase2 knockdown, indicating a cytoprotective role for nSMase2 (Fig. 7b). nSMase2 is highly expressed in the brain and bones $^{20}$. Presently, nSMase2 was highly expressed in mouse striatum and midbrain, including the substantia nigra region, as verified by the enrichment of the dopaminergic neuron markers tyrosine hydroxylase and dopamine transporter (Fig. 7c). This finding prompted us to examine dopaminergic stress-induced cytotoxicity. Because mitochondrial dysfunction plays a central role in pathogenesis of $\mathrm{PD}^{45}$, the cytotoxicity induced by the mitochondrial uncoupler carbonyl cyanide mchlorophenyl hydrazine (CCCP) was tested. nSMase2 knockdown significantly increased CCCP-induced cytotoxicity (Fig. 7d). Furthermore, CCCP-induced autophagic flux was attenuated by nSMase 2 knockdown or inhibition (Supplementary Figures 6A and 6B), demonstrating that nSMase2 contributes to CCCP-induced autophagy. In addition to mitochondrial dysfunction, oxidative stress and protein adducts caused by dopamine oxidation can contribute to the progression of $\mathrm{PD}^{46}$. Thus, we examined the effect of a high dose of dopamine exposure. Consistent with the CCCP treatment results, nSMase 2 knockdown enhanced the cell death induced by the high dose of dopamine (Supplementary Figure 6C). The results indicate the protective role of $n$ SMase 2 against dopaminergic toxicity.

To confirm whether the cell-protective role of nSMase2 depends on autophagy regulation, cytotoxicity was determined in the presence or absence of the autophagy inhibitor CQ. There were no significant differences in starvation or CCCP-induced cytotoxicity between siControl and siSmpd3 transfected cells in the presence of CQ, demonstrating that autophagy inhibition is epistatic to nSMase2 repression in cell death (Fig. 7e). Thus, the cytoprotective role of nSMase 2 depends on autophagy. Treatment with CQ enhanced cell death induced by starvation or CCCP treatment in control siRNA transfected cells (Fig. 7e), also implying that cells experiencing the toxic stress activate the autophagy process as a protective mechanism.

One of the major risk factors for PD is aging, which may reduce the autophagic function. Thus, we examined the level of nSMase 2 in the brain, especially in the striatum, of old and young mice. As expected, the level of nSMase 2 expression was lower and p62 accumulation was higher in old mice than in young mice (Fig. 7f). Furthermore, we analyzed the correlation between nSMase 2 and PD using a GEO database (GSE7621) of human substantia nigra. Interestingly, SMPD3 expression in the patient group was significantly lower than in the healthy group (Fig. $7 \mathrm{~g}$ ). The expression of some autophagy-associated genes was also reduced in patients with $\mathrm{PD}$, and significant correlations between SMPD3 and ATG genes, such as $A T G 9 B$ and ATG10, were observed (Fig. 7g).

The collective findings demonstrate the nSMase2mediated induction of cytoprotective autophagy via p38 MAPK activation and mTOR suppression (Fig. 8), and strongly suggest the preventive or therapeutic role of nSMase2 for PD through autophagy induction.

\section{Discussion}

Autophagic processes are closely associated with membrane dynamics ${ }^{1,5}$. Ceramides play central roles in 


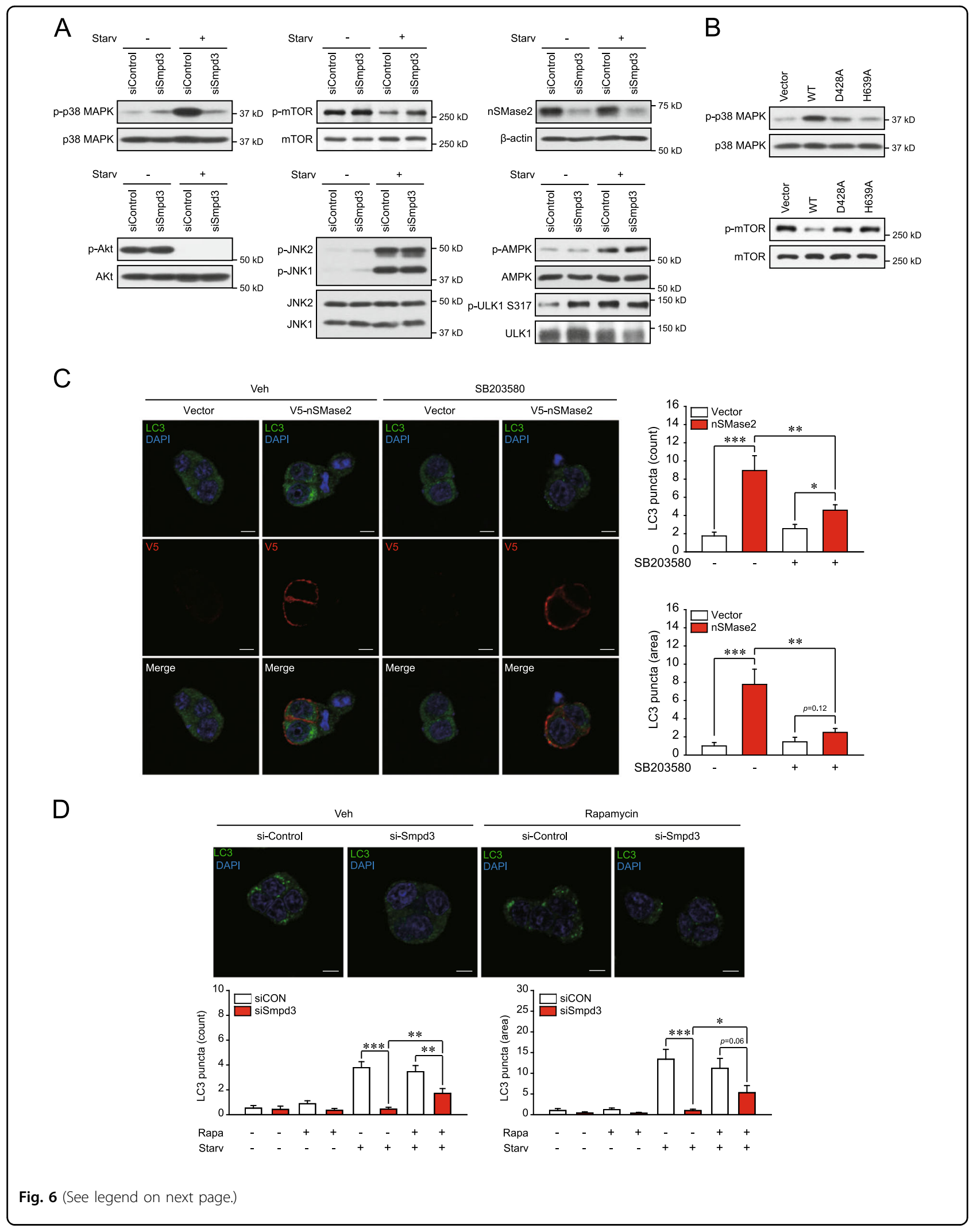


(see figure on previous page)

Fig. 6 nSMase2 induces autophagy via p38 MAPK activation and mTOR inhibition. a Suppression of starvation-induced p38 MAPK

phosphorylation and starvation-reduced mTOR phosphorylation by knockdown of nSMase2. PC12 cells were transfected with 50 nM nSMase2 siRNA (siSmpd3) or non-targeting negative control siRNA (siControl). At $48 \mathrm{~h}$ after transfection, the cells were incubated with HBSS for $2 \mathrm{~h}$. Phospho-p38 MAPK, p38 MAPK, phosphor-mTOR, mTOR, phospho-Akt, Akt, phospho-JNK1/2, JNK1/2, phospho-AMPK, AMPK, phospho-ULK1 (Ser317), and ULK1 levels were analyzed by immunoblotting, and nSMase2 knockdown was confirmed simultaneously by immunoblotting. b Enhanced p38 MAPK phosphorylation and suppressed mTOR phosphorylation by nSMase2. PC12 cells were transfected with either plasmid-encoding V5-tagged WT nSMase2, catalytically inactive forms of nSMase2 (D428A and H639A), or empty vector. At $48 \mathrm{~h}$ after transfection, phospho-p38 MAPK, p38 MAPK, phospho-mTOR, and mTOR levels were analyzed by immunoblotting. c p38 MAPK inhibition with SB203580 attenuated the autophagy induction by nSMase2 overexpression. Cells overexpressing V5-tagged nSMase2 were pretreated with $10 \mu M$ SB203580 or the vehicle for $3 \mathrm{~h}$. LC 3 puncta were detected by immunofluorescence assay using anti-LC3 antibody and counted using ImageJ software in V5-positive cells. Scale bar $=5 \mu \mathrm{m}$. $\mathbf{d}$ mTOR inhibition with rapamycin partially reversed the effect of nSMase2 knockdown on LC3 puncta formation. PC12 cells transfected with siRNA (as described above) were pretreated with $750 \mathrm{nM}$ rapamycin or the vehicle for $1 \mathrm{~h}$ and then starved for $2 \mathrm{~h}$. LC3 puncta were detected by immunofluorescence assay using anti-LC3 antibody and counted using ImageJ software. Representative images of siRNA-transfected cells under starvation conditions are provided. Scale bar $=5 \mu \mathrm{m}$; Significant differences between the indicated groups, ${ }^{*} p<0.05,{ }^{* *} p<0.01$, and ${ }^{* * *} p<0.001$

sphingolipid metabolism as membrane components that mediate membrane dynamics by altering fluidity or rigidity, and can also function as signaling molecules ${ }^{6}$. In this context, several studies have revealed that treatment with exogenous ceramides can induce autophagy ${ }^{16-18,47}$. Recently, the de novo pathway of ceramide synthesis was suggested to be involved in melatonin-induced autophagic cell death ${ }^{48}$ and sodium selenite-induced lethal mitophagy ${ }^{49}$. In contrast, this study showed that in a nutrient starvation model, arguably the most physiologically relevant stimulus of autophagy ${ }^{50}$, the de novo pathway of ceramide synthesis had negligible effect on autophagic flux (Fig. 1). Moreover, nSMase2 knockdown or inhibition with a specific inhibitor significantly suppressed starvation-induced autophagic flux (Figs. 1, 2), suggesting that nSMase2 mediates starvation-induced autophagy.

Increased serine phosphorylation of nSMase2 can enhance protein stability ${ }^{30}$. Consistent with this, we found that under nutrient-starvation conditions, nSMase 2 protein level and activity rapidly and stably increased within 15-30 min. nSMase2 was phosphorylated at serine residues within 15 min after starvation, with no change in mRNA levels, with subsequent LC3 turnover and p62 degradation within 60-120 min (Fig. 3). This suggests that activation of nSMase2 can lead to the induction of autophagy. We also found that treatment with cyclosporine A (CsA), a PP2B inhibitor, had a similar effect on autophagy as the overexpression of nSMase2 (Supplementary Figure 7). A study on cardiomyocytes also supported our finding ${ }^{51}$. Thus, nutrient starvation may inhibit dephosphorylation of nSMase2 by PP2B, thereby inducing nSMase2-mediated autophagy.

In particular, the Golgi-localized ceramides induced by starvation (Fig. 4) and the diminished autophagy by CERT inhibitor that limited ceramide transport from the ER to the Golgi complex, which was recovered by ceramide supply (Fig. 5), suggest that localization of ceramides to the Golgi complex is the key step of autophagy regulation, with respect to the flow of ceramides. Inhibition of CERT and SMS, which are the upstream molecules of nSMase2, suppressed autophagy, while the inhibition of de novo synthesis upstream of CERT did not affect autophagy during starvation. The reason might be the sufficient basal level of ceramide in ER. The markedly greater level of ceramides in ER than in the Golgi apparatus ${ }^{52}$ indicates that the level of Golgi-localized ceramides reply mainly on the direct supply to the Golgi, rather than on the de novo synthesis of ceramide in the ER.

To elucidate the mechanism of starvation-induced autophagy via the activation of the nSMase2-ceramide pathway in the Golgi complex, we examined the correlation between activation of this pathway and several regulatory pathways of autophagy. In one study, p38 MAPK mediated starvation-induced autophagy in mouse embryonic fibroblasts ${ }^{39}$. However, the role of p38 MAPK in autophagy remains largely unknown. Consistent with the previous study, we found that starvation strongly induced phosphorylation of p38 MAPK (Fig. 6a). While p38 MAPK can activate nSMase $2^{53,54}$, our results instead showed that nSMase2 can mediate the phosphorylation of p38 MAPK induced by starvation (Fig. 6a). This phosphorylation was significantly reduced by knockdown or enzymatic inactivation of nSMase2. Moreover, treatment with the p38 MAPK inhibitor, SB203580, diminished nSMase2 overexpression-induced LC3 puncta formation and p62 degradation (Fig. 6c and Supplementary Figure $5 \mathrm{C}$ ), suggesting that nSMase2-induced phosphorylation of p38 MAPK explains their sequential roles in autophagy.

Our data also suggest that nSMase 2 inhibition of mTOR is involved in nSMase2-dependent autophagy induction during starvation (Fig. 6a-d). Inhibition of Akt may be the cause of mTOR inhibition by nSMase2, because ceramides inhibit Akt, and Akt activates $\mathrm{mTOR}^{40,41}$; however, Akt phosphorylation was not affected by knockdown of nSMase2 (Fig. 6a), indicating that the inhibition of 





(see figure on previous page)

Fig. 7 Cytoprotective role of nSMase2 against starvation and CCCP exposure depending on autophagy, and its relationship with PD. a Prosurvival effect of nSMase2 against starvation. PC12 cells were transfected with $50 \mathrm{nM}$ nSMase2 siRNA (siSmpd3) or non-targeting negative control siRNA (siCON). At $48 \mathrm{~h}$ after transfection, cells were incubated with HBSS for 7 or $10 \mathrm{~h}$. Levels of lactate dehydrogenase (LDH) released into the medium and total LDH were quantified to calculate cell cytotoxicity. The data represent the mean \pm SEM of three independent experiments. $\mathbf{b}$ Images of dead cells. PC12 cells transfected with siRNA were starved for $10 \mathrm{~h}$ and then double-stained with PI and Hoechst 33342. Dead cells were positive for PI, and total cells were labeled with Hoechst 33342. Pl positive cells were counted for more than 800 cells per group. Scale bar $=100 \mu \mathrm{m}$ c High expression of nSMase2 in the dopaminergic neuron-enriched area. Expression level of nSMase2 was detected in the mouse brain by immunoblotting. $\mathbf{d}$ Cytoprotective effect of nSMase2 against CCCP exposure. PC12 cells transfected with siRNA were treated with 20 or $40 \mu \mathrm{M}$ CCCP for $24 \mathrm{~h}$. e Autophagy-dependent cytoprotective role of nSMase2. The cells transfected with indicated siRNA were starved for $7 \mathrm{~h}$ or treated with 20 $\mu \mathrm{M}$ CCCP for $24 \mathrm{~h}$ in the presence or absence of $50 \mu \mathrm{M}$ CQ. Cytotoxicity was determined by the LDH assay. The data represent the mean \pm SEM of three independent experiments. $\mathbf{f}$ Reduced levels of nSMase 2 in the striatum of old mice with p62 accumulation. Expression levels of nSMase2 and p62 in young ( 6 weeks) and old (20 months) mice were assessed by immunoblotting and normalized to $\beta$-actin levels. $\mathbf{g}$ Decreased expression of nSMase2 and autophagy-associated genes in the substantia nigra of patients with PD (upper). Positive correlation between nSMase2 and ATGs (lower). Data were extracted from GSE7621 data set (Control, $n=9$; PD patients, $n=16$ )

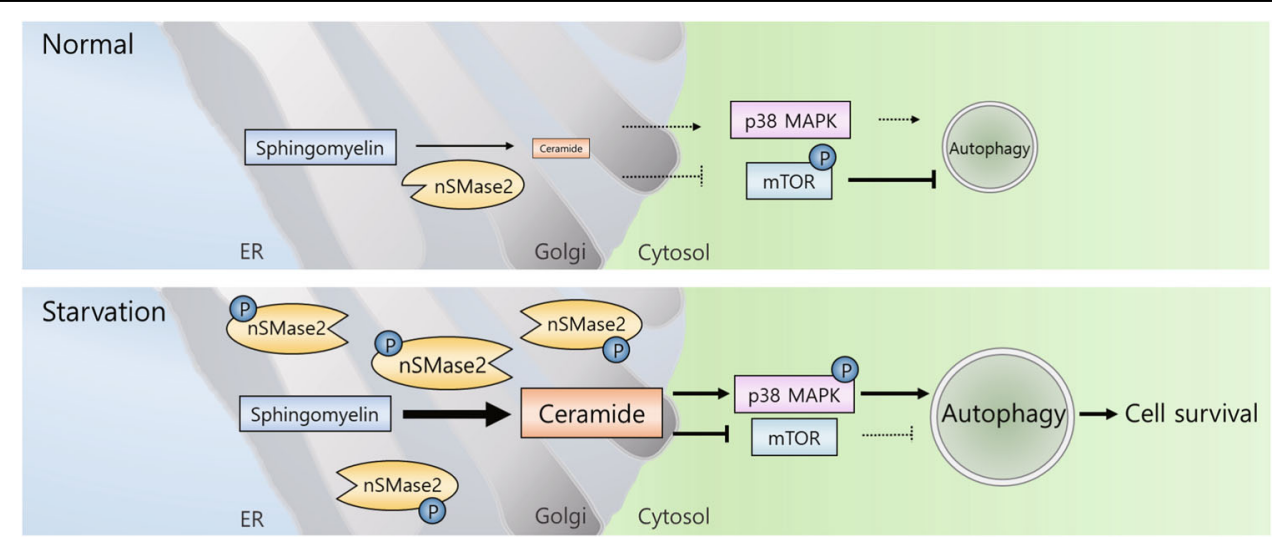

Fig. 8 Working model for the nSMase2-mediated induction of cytoprotective autophagy during starvation. Under normal conditions, autophagy is suppressed by phosphorylated mTOR. During nutrient starvation, upregulated nSMase2 produces ceramides in the Golgi that activate p38 MAPK and inhibits mTOR signaling, thereby inducing autophagic flux

mTOR cannot be explained by ceramide-induced inhibition of Akt. Similarly, ceramides can activate the JNKbeclin 1 pathway ${ }^{16}$. However, nSMase2 knockdown did not affect JNK (Fig. 6a). Although the effect of ceramide on AMPK is still controversial, ceramide-mediated induction of AMPK phosphorylation was described ${ }^{42}$. However, starvation-induced phosphorylation of AMPK and its downstream target ULK1 at serine 317 were not reduced by nSMase2 knockdown (Fig. 6a). Since the increased phosphorylation of ULK1 at the serine 317 residue by nSMase 2 knockdown occurred only in the basal condition without starvation and was not accompanied by AMPK activation, the effect of nSMase2 knockdown on ULK1 was not studied further, which would be elucidated by later studies. The precise mechanism by which nSMase2-derived ceramides in the Golgi complex regulate p38MAPK and mTOR as key effector molecules in autophagic processes also remains to be elucidated. Nevertheless, these results indicate that ceramides can mediate autophagy through starvation- induced activation of nSMase2, and is related to both $\mathrm{p} 38$ MAPK activation and mTOR inhibition as a possible signaling molecule.

Additionally, the mechanical role of ceramides in membrane dynamics may be a possible mechanism of nSMase2mediated induction of autophagy. This needs to be investigated. Conversion of SM to ceramides by bacterial SMase induces lipid membrane modifications, such as vesicle collapse and rupture ${ }^{55}$. Moreover, in mammalian cells, nSMase2 deficiency disturbs the Golgi secretory pathway ${ }^{56}$ and exosome budding into multivesicular endosomes ${ }^{23,24}$. During autophagy, cellular organelles including the Golgi apparatus $^{1,57}$ participate in autophagosome formation by providing membrane sources. The inhibition of membrane supply from the Golgi results in failure of vesicle formation during autophagy ${ }^{58}$. Further investigation regarding nSMase2 regulation of Golgi membrane dynamics, such as budding to provide membrane sources for autophagosomes, may contribute to a better understanding of how nSMase2 induces autophagic flux. 
Autophagy primarily plays a vital role in maintaining cellular homeostasis that prolongs cell survival, but lethal autophagy also exists. Lethal autophagy is induced by exogenous ceramide ${ }^{16,17}$ and $\mathrm{CerS}^{48,49}$. In contrast, nSMase2 can be cytoprotective during starvation (Fig. 7a, b), implying that the survival or lethal outcome of ceramide-induced autophagy may depend on its subcellular localization and role in signaling and/or membrane dynamics. Highly secretory cells, such as neurons, may be more dependent on cytoprotective autophagy because of the accumulation of higher levels of misfolded proteins and aggregates following abundant protein synthesis. Protein aggregation is a common feature of various neurodegenerative diseases, including $\mathrm{PD}$ and $\mathrm{AD}^{4}$, and depletion of $A T G$ causes neurodegeneration ${ }^{59}$. nSMase2 is highly expressed in brain ${ }^{9}$. In the brain, nSMase2 was abundant in the striatum and midbrain (Fig. 7c) containing dopaminergic neurons which are lost in $\mathrm{PD}^{60}$. nSMase2 also protected cells against dopaminergic stressors (Fig. 7d and Supplementary Figure 6C). The cytoprotective role of nSMase 2 may depend on autophagy (Fig. 7e).

In addition, as shown in Fig. $7 \mathrm{f}, \mathrm{g}$, nSMase 2 gene expression in the substantia nigra of patients with PD was significantly reduced compared with healthy controls, and protein expression of nSMase 2 in the mouse striatum decreased with aging-a major risk factor for $\mathrm{PD}^{61}$. Consistent with our finding, in a neurodegeneration model of Drosophila with defects in autophagy, neutral SMase overexpression rescued neurodegeneration ${ }^{62}$. Ciliogenesis and differentiation for neural development mediated by nSMase 2 were reported in human stem cells and neural progenitor cells ${ }^{63}$. On the other hand, nSMase2 deficiency influenced recovery the 5XFAD mouse model of early onset AD with amyloid- $\beta$ pathol$\mathrm{ogy}^{64}$. However, the model does not seem to fully reflect human $\mathrm{AD}$ because of the lack of taupathy ${ }^{65}$, which is another major pathological hallmark and a more potent target of $\mathrm{AD}$ in human clinical trials ${ }^{66}$. In addition, in contrast to other $\mathrm{AD}$ models including the human tau transgenic mouse model, in which autophagy activation alleviates the disease state ${ }^{67,68}$, the inhibition of autophagy can ameliorate $\mathrm{AD}$ pathology in the 5XFAD model ${ }^{69}$. Therefore, the effect of nSMase 2 modulation in other AD models, including the taupathy model, could provide clearer understanding of its action on AD. Indeed, inhibition of nSMase2 disrupts synaptic plasticity and memory in C57BL/6 mice ${ }^{70}$.

This study demonstrates that nSMase2 can mediate starvation-induced autophagy and that activation of nSMase2 is essential for autophagy induction. This suggests that the nSMase2-Golgi-localized ceramide pathway plays a critical role in autophagy, providing insights into the mechanisms underlying human diseases.

\section{Experimental procedures \\ Reagents}

RPMI-1640 medium, horse serum (HS), fetal bovine serum (FBS), HBSS, phosphate-buffered saline (PBS), penicillin/streptomycin, Lipofectamine RNAiMAX, Lipofectamine 2000, 4',6-diamidino-2-phenylindole (DAPI), ProLong Gold, Alexa-conjugated secondary antibodies (Alexa Fluor 488-conjugated goat anti-rabbit IgG, A11008; Alexa Fluor 594-conjugated goat anti-mouse IgM, A-21044; Alexa Fluor 594-conjugated goat antimouse IgG, A-11032), and anti-V5 antibody (\#46-0705) were purchased from Thermo Fisher Scientific (Waltham, MA, USA). Poly-D-lysine-coated 6-well plates, 24-well plates, and 100-mm dishes were obtained from Corning (Corning, NY, USA). Poly-D-lysine-coated coverslips (GG-12-PDL) were supplied by Neuvitro (Vancouver, WA, USA). Desipramine, FB1, and SB203580 were obtained from Tocris Bioscience (Bristol, UK). GW4869, myriocin, and anti-ULK1 antibody (A7481) were purchased from Sigma-Aldrich (St. Louis, MO, USA). HPA12 was supplied by Tokyo Chemical Industry (Tokyo, Japan). Protease inhibitor cocktail and PhosSTOP phosphatase inhibitor cocktail were provided by Roche (Basel, Switzerland). Bovine serum albumin (BSA) was purchased from Bioworld (Dublin, OH, USA). Anti-LC3B (NB1002220) and anti-p62 antibodies (H00008878-M01) were obtained from Novous Biologicals (Littleton, CO, USA). C2-ceramide, C6-ceramide, anti-ceramide mouse IgM (ALX-804-196-T050), and anti-calnexin antibody (ADISPA-860-D) were provided by Enzo Life Sciences (Farmingdale, NY, USA). Anti-giantin (ab24586) antibody was obtained from Abcam (Cambridge, UK). Antiphospho-p38 MAPK (Thr180/Tyr182; \#4631), anti-p38 MAPK (\#9212), anti-phospho-mTOR (Ser2448; \#2971), anti-mTOR (\#2972), anti-phospho-Akt (Ser473; \#4058), anti-Akt(\#4685), anti-phospho-JNK1/2 (Thr183/Tyr185; \#4668), anti-JNK1/2 (\#9252), anti-phospho-AMPKa (Thr172; \#2535), anti-AMPKa (\#2532), anti-phosphoULK1 (Ser317; \#12753), and horseradish peroxidase (HRP)-conjugated secondary antibodies (anti-rabbit IgG, \#7074; anti-mouse IgG, \#7076) were purchased from Cell Signaling Technology (Danvers, MA, USA). AntinSMase2 (sc-166637 for immunoblot analysis; sc-67305 for immunofluorescence analysis), anti-tyrosine hydroxylase (sc-14007), anti-glyceraldehyde 3-phosphate dehydrogenase (GAPDH, sc-25778), and anti- $\beta$-actin (sc47778) antibodies were provided by Santa Cruz Biotechnology (Santa Cruz, CA, USA). Anti-phosphoserine (AB1603) and anti-dopamine transporter (MAB369) antibodies were obtained from EMD Millipore (Billerica, MA, USA). Dimethyl sulfoxide (DMSO) was supplied by Duchefa Biochemie (Haarlem, Netherlands). Unless otherwise stated, all other reagents were purchased from Sigma-Aldrich. 


\section{Cell culture and treatment}

Rat neuroblastoma PC12 cell line was purchased from American Type Culture Collection (Manassas, VA, USA). PC12 cells were cultured in RPMI-1640 medium containing $10 \% \mathrm{HS}$ and $5 \% \mathrm{FBS}$ at $37^{\circ} \mathrm{C}$ in a humidified atmosphere of $5 \% \mathrm{CO}_{2}$. For nutrient starvation, cells were incubated with HBSS for $2 \mathrm{~h}$ in the presence or absence of $50 \mu \mathrm{M}$ CQ after washing with HBSS. For inhibitor studies, the indicated concentration of each inhibitor or vehicle was added to cells $1 \mathrm{~h}$ prior to and during the starvation period.

\section{Transfection with siRNA or plasmid}

For nSMase2 knockout, a pool of two siRNAs against rat Smpd3 (RSS331830 and RSS331831, Stealth siRNA, Thermo Fisher Scientific) was used throughout the study with negative control siRNA (\#12935-300) as a control. ON-TARGETplus SMART pool siRNA of rat Smpd3 (L095934-02; Dharmacon) and its negative control siRNA (D-001810-10) were additionally used in the experiment confirming the effect of nSMase2 knockdown on autophagy. PC12 cells were transfected with $50 \mathrm{nM}$ of the indicated siRNA using Lipofectamine RNAiMAX. For overexpression of V5-tagged nSMase2, cells were transfected with pcDNA3.1/V5-His-TOPO vector encoding murine Smpd3 using Lipofectamine 2000 following manufacturer's instructions. The vector was a gift from Dr. Yusuf Hannun. Cells were used for further experiments at $48 \mathrm{~h}$ post-transfection.

\section{Immunoblot analysis}

Cells were homogenized by sonication in radioimmunoprecipitation assay (RIPA) buffer (Bioworld) containing protease inhibitor cocktail and PhosSTOP phosphatase inhibitor cocktail. The lysates were centrifuged at $10,000 \times g$ at $4{ }^{\circ} \mathrm{C}$ for $10 \mathrm{~min}$, and the supernatants were collected. Proteins in the lysates were separated by sodium dodecyl sulfate-polyacrylamide gel electrophoresis (SDS-PAGE) and transferred to a polyvinylidene fluoride membrane (Bio-Rad Laboratories, Hercules, CA, USA). The membranes were blocked for 1 $h$ using Tris-buffered saline (TBS) containing $5 \%$ BSA and $0.1 \%$ Tween-20, and then incubated with primary antibodies against target proteins in 5\% BSA and $0.1 \%$ Tween-20 in TBS for $24 \mathrm{~h}$ at $4{ }^{\circ} \mathrm{C}$. After three washes with $0.1 \%$ Tween-20 in TBS, the membranes were incubated with HRP-conjugated secondary antibodies in 5\% BSA and $0.1 \%$ Tween-20 in TBS for $1 \mathrm{~h}$ at room temperature (RT). Proteins were detected using the chemical reaction of HRP with electrochemiluminescence (ECL) substrates (GenDEPOT, Katy, TX, USA).

\section{Immunofluorescence assay}

Cells were fixed with $4 \%$ formaldehyde in PBS for 20 min. Fixed cells were washed three times with $0.3 \%$ Triton
X-100 (TX-100) in PBS and incubated with 5\% BSA and $0.3 \% \mathrm{TX}-100$ in PBS for $1 \mathrm{~h}$ at RT. The cells were then incubated with primary antibodies (anti-ceramide, 1:10; anti-nSMase2, 1:100; anti-LC3, 1:300; anti-V5, 1:500; antigiantin, 1:500; anti-calnexin, 1:500) against target proteins in $1 \% \mathrm{BSA}$ and $0.3 \% \mathrm{TX}-100$ in PBS for $24 \mathrm{~h}$ at $4{ }^{\circ} \mathrm{C}$. After three washes, cells were incubated with Alexa-conjugated secondary antibodies (1:350) for $1 \mathrm{~h}$ at RT. DAPI was used for nuclear staining and coverslips were mounted with ProLong Gold antifade reagent after three washes. Cell images were acquired with a confocal microscope (LSM 710; Zeiss, Oberkochen, Germany). LC3-positive puncta numbers and size were measured with the GFP-LC3 macro $^{71}$ using ImageJ software $(\mathrm{NIH}$, Bethesda, MD, USA) for more than 15 cells per group.

\section{nSMase 2 activity assay}

nSMase 2 activities of $10 \mu \mathrm{g}$ of cell homogenates were assessed using $\left[{ }^{14} \mathrm{C}\right]$-sphingomyelin as a substrate in a mixture with $100 \mathrm{mM}$ Tris- $\mathrm{HCl}$ ( $\mathrm{pH}$ 7.5) buffer containing $0.1 \% \mathrm{TX}-100,10 \mathrm{mM} \mathrm{MgCl}_{2}, 5 \mathrm{mM}$ dithiothreitol, and $50 \mu \mathrm{M}$ phosphatidylserine as described previously ${ }^{21}$. The reaction proceeded for $20 \mathrm{~min}$ at $37^{\circ} \mathrm{C}$ and terminated by adding chloroform/methanol/2N $\mathrm{HCl}$ (1:1:0.1, v/v). Samples were centrifuged for phase separation, and the radioactivity of the upper phase was determined by liquid scintillation counting (MicroBeta2; PerkinElmer, Waltham, MA).

\section{Pull-down of nSMase2}

For pull-down of nSMase2, $30 \mu \mathrm{g}$ of cell lysates in $100 \mathrm{mM}$ Tris- $\mathrm{HCl}$ (pH 7.5) buffer containing $0.1 \%$ Triton X-100 and $10 \mathrm{mM} \mathrm{MgCl}_{2}$ were incubated with $10 \mu \mathrm{M}$ biotin-conjugated SM (Echelon, S-400B) on a rocker for $2 \mathrm{~h}$ at $4{ }^{\circ} \mathrm{C}$. Sequentially, streptavidin-Sepharose beads (GE Healthcare, Buckinghamshire, UK, \#71-5004-40 AE) was added to the samples and incubated on a rocker overnight at $4{ }^{\circ} \mathrm{C}$. The samples were centrifuged at $700 \times g$ at $4{ }^{\circ} \mathrm{C}$ for $1 \mathrm{~min}$, and the collected pellets were washed five times with cold PBS. After washing, the pellets were treated with SDS sample buffer and continuously analyzed by immunoblotting.

\section{Detection of mRNA levels}

RNA was extracted from PC12 cells using TRIzol reagent (Invitrogen, Carlsbad, CA, USA). For cDNA synthesis, the extracted RNA was reverse transcribed using a SuperScript III First-Strand Synthesis System (Invitrogen). Quantitative real-time polymerase chain reaction (PCR) was performed using SYBR Green Supermix (Bio-Rad Laboratories) and detected by the CFX Connect ${ }^{\text {tw }}$ real-time PCR detection system (Bio-Rad Laboratories) according to the manufacturer's instructions. mRNA expression was normalized to Hprt1 mRNA levels. 
To confirm the expression of ceramide-producing enzymes in PC12 cells, extracted mRNA was analyzed by reverse transcription PCR (RT-PCR). After cDNA synthesis was performed as described above, Maxime iTaq PCR PreMix (iNtRON Biotechnology, Seongnam, Republic of Korea) was used for PCR. PCR products were detected using the ChemiDoc imaging system (Bio-Rad Laboratories) after PAGE. All PCR primers used in this study are listed in Supplementary Table 1.

\section{LDH assay}

Cell cytotoxicity was determined by LDH release using the CytoTox $96^{\circ}$ Non-Radioactive Cytotoxicity Assay (G1780; Promega, Madison, WI, USA) according to the manufacturer's instructions. Cells cultured in poly-Dlysine-coated 24 -well plates $\left(1.5 \times 10^{5}\right.$ cells/well $)$ were subjected to nutrient starvation and treated with the indicated concentrations of dopamine or CCCP. After the designated treatment times, culture supernatants were collected and incubated with the reaction mixture to measure LDH activity. LDH released into the medium was calculated as the percentage of total LDH.

\section{PI/Hoechst 33342 stain}

To image dead cells, PC12 cells were seeded on poly-Dlysine-coated coverslips $\left(1.5 \times 10^{5}\right.$ cells/well $)$ and cultured in growth medium for 1 day. Cells under the indicated stress conditions were incubated with $50 \mathrm{ng} / \mathrm{mL}$ PI (BD Biosciences, \#51-66211E) and $10 \mu \mathrm{g} / \mathrm{mL}$ Hoechst 33342 dye (Thermo Fisher Scientific, H3570) for 30 min. Images of cells stained with blue-fluorescent Hoechst 33342 and red-fluorescent PI were obtained by a Ni-U fluorescence microscope (Nikon, Tokyo, Japan) equipped with a digital camera (DS-Ri1; Nikon).

\section{Brain dissection}

Young (6 weeks) and old ( 20 months) male C57BL/6 mice were obtained from the Korea Research Institute of Bioscience and Biotechnology (Daejeon, Korea). All animal experiments were performed in accordance with the National Research Council's Guidelines for the Care and Use of Laboratory Animals and Guidelines for Animal Experiments of Chung-Ang University, and were approved by the University Committee for Animal Experiments (approval no: 2014-00031). Mice brains were dissected according to a previously described method ${ }^{72}$, and the brain tissues were analyzed by immunoblotting.

\section{Statistical analysis}

All data are expressed as the mean \pm standard error of the mean (SEM). Unless otherwise stated, differences between two groups were analyzed using the Student's $t$ test. In all cases, a p-value $<0.05$ was considered statistically significant.

\section{Acknowledgements}

The authors thank Dr. Yusuf Hannun for kindly providing plasmid-encoding V5tagged nSMase2. This research was supported by the National Research Foundation of Korea (NRF) grant funded by the Ministry of Science, ICT \& Future Planning (NRF-2015M3A9C7030121).

\section{Conflict of interest}

The authors declare that they have no conflict of interest

\section{Publisher's note}

Springer Nature remains neutral with regard to jurisdictional claims in published maps and institutional affiliations.

Supplementary Information accompanies this paper at https://doi.org/ 10.1038/s41419-018-0709-4.

Received: 22 December 2017 Revised: 18 April 2018 Accepted: 2 May 2018 Published online: 04 June 2018

\section{References}

1. Shibutani, S. T. \& Yoshimori, T. A current perspective of autophagosome biogenesis. Cell Res. 24, 58-68 (2014).

2. Mizushima, N. Autophagy: process and function. Genes Dev. 21, 2861-2873 (2007).

3. Levine, B. \& Kroemer, G. Autophagy in the pathogenesis of disease. Cell 132, 27-42 (2008).

4. Ross, C. A. \& Poirier, M. A. Protein aggregation and neurodegenerative disease. Nat. Med. 10(Suppl), S10-S17 (2004).

5. Carlsson, S. R. \& Simonsen, A. Membrane dynamics in autophagosome biogenesis. J. Cell. Sci. 128, 193-205 (2015).

6. van Blitterswijk, W. J., van der Luit, A. H., Veldman, R. J., Verheij, M. \& Borst, J. Ceramide: second messenger or modulator of membrane structure and dynamics? Biochem. J. 369, 199-211 (2003).

7. Hirschberg, K., Rodger, J. \& Futerman, A. H. The long-chain sphingoid base of sphingolipids is acylated at the cytosolic surface of the endoplasmic reticulum in rat liver. Biochem. J. 290(Pt 3), 751-757 (1993).

8. Milhas, D., Clarke, C. J., Idkowiak-Baldys, J., Canals, D. \& Hannun, Y. A. Anterograde and retrograde transport of neutral sphingomyelinase-2 between the Golgi and the plasma membrane. Biochim. Biophys. Acta 1801, 1361-1374 (2010).

9. Hofmann, K., Tomiuk, S., Wolff, G. \& Stoffel, W. Cloning and characterization of the mammalian brain-specific, Mg2+-dependent neutral sphingomyelinase. Proc. . Natl. Acad. Sci. USA 97, 5895-5900 (2000).

10. Schissel, S. L., Schuchman, E. H., Williams, K. J. \& Tabas, I. Zn2+-stimulated sphingomyelinase is secreted by many cell types and is a product of the acid sphingomyelinase gene. J. Biol. Chem. 271, 18431-18436 (1996).

11. Auge, N. et al. The sphingomyelin-ceramide signaling pathway is involved in oxidized low density lipoprotein-induced cell proliferation. J. Biol. Chem. 271, 19251-19255 (1996)

12. Imgrund, S. et al. Adult ceramide synthase 2 (CERS2)-deficient mice exhibit myelin sheath defects, cerebellar degeneration, and hepatocarcinomas. J. Biol. Chem. 284, 33549-33560 (2009).

13. Maupas-Schwalm, F. et al. The sphingomyelin/ceramide pathway is involved in ERK1/2 phosphorylation, cell proliferation, and UPAR overexpression induced by tissue-type plasminogen activator. FASEB J.: Off. Publ. Fed. Am. Soc. Exp. Biol. 18, 1398-1400 (2004)

14. Boppana, N. B. et al. Ceramide synthase inhibitor fumonisin B1 inhibits apoptotic cell death in SCC17B human head and neck squamous carcinoma cells after Pc4 photosensitization. Photochem. \& Photobiol. Sci.: Off. J. Eur. Photochem. Assoc. Eur. Soc. Photobiol. 13, 1621-1627 (2014).

15. Separovic, D. et al. siRNA-mediated down-regulation of ceramide synthase 1 leads to apoptotic resistance in human head and neck squamous carcinoma cells after photodynamic therapy. Anticancer Res. 32, 2479-2485 (2012).

16. Li, D. D. et al. The pivotal role of C-Jun NH2-terminal kinase-mediated Beclin 1 expression during anticancer agents-induced autophagy in cancer cells. Oncogene 28, 886-898 (2009). 
17. Daido, S. et al. Pivotal role of the cell death factor BNIP3 in ceramide-induced autophagic cell death in malignant glioma cells. Cancer Res. 64, 4286-4293 (2004).

18. Scarlatti, F. et al. Ceramide-mediated macroautophagy involves inhibition of protein kinase B and up-regulation of beclin 1. J. Biol. Chem. 279, 18384-18391 (2004).

19. Shamseddine, A. A., Airola, M. V. \& Hannun, Y. A. Roles and regulation of neutral sphingomyelinase-2 in cellular and pathological processes. Adv. Biol. Regul. 57, 24-41 (2015).

20. Khavandgar, $Z$. et al. A cell-autonomous requirement for neutral sphingomyelinase 2 in bone mineralization. J. Cell. Biol. 194, 277-289 (2011).

21. Marchesini, $\mathrm{N}$. et al. Role for mammalian neutral sphingomyelinase 2 in confluence-induced growth arrest of MCF7 cells. J. Biol. Chem. 279, 25101-25111 (2004).

22. Stoffel, W., Jenke, B., Block, B., Zumbansen, M. \& Koebke, J. Neutral sphingomyelinase 2 (smpd3) in the control of postnatal growth and development. Proc. Natl. Acad. Sci. USA 102, 4554-4559 (2005).

23. Trajkovic, K. et al. Ceramide triggers budding of exosome vesicles into multivesicular endosomes. Science (New York, NY) 319, 1244-1247 (2008).

24. Kosaka, N. et al. Neutral sphingomyelinase 2 (nSMase2)-dependent exosomal transfer of angiogenic microRNAs regulate cancer cell metastasis. J. Biol. Chem. 288, 10849-10859 (2013).

25. Mizushima, N., Yoshimori, T. \& Levine, B. Methods in mammalian autophagy research. Cell 140, 313-326 (2010).

26. Lee, J. K. et al. Acid sphingomyelinase modulates the autophagic process by controlling lysosomal biogenesis in Alzheimer's disease. J. Exp. Med. 211, 1551-1570 (2014).

27. Fucho, R. et al. ASMase regulates autophagy and lysosomal membrane permeabilization and its inhibition prevents early stage non-alcoholic steatohepatitis. J. Hepatol. 61, 1126-1134 (2014).

28. Clarke, C. J., Cloessner, E. A., Roddy, P. L. \& Hannun, Y. A. Neutral sphingomyelinase 2 (nSMase2) is the primary neutral sphingomyelinase isoform activated by tumour necrosis factor-alpha in MCF-7 cells. Biochem. J. 435, 381-390 (2011).

29. Luberto, C. et al. Inhibition of tumor necrosis factor-induced cell death in MCF7 by a novel inhibitor of neutral sphingomyelinase. J. Biol. Chem. 277, 41128-41139 (2002)

30. Filosto, S., Ashfaq, M., Chung, S., Fry, W. \& Goldkorn, T. Neutral sphingomyelinase 2 activity and protein stability are modulated by phosphorylation of five conserved serines. J. Biol. Chem. 287, 514-522 (2012).

31. Hanada, K. Intracellular trafficking of ceramide by ceramide transfer protein. Proc. Jpn. Acad. Ser. B Phys. Biol. Sci. 86, 426-437 (2010).

32. Fukasawa, M., Nishijima, M. \& Hanada, K. Genetic evidence for ATP-dependent endoplasmic reticulum-to-Golgi apparatus trafficking of ceramide for sphingomyelin synthesis in Chinese hamster ovary cells. J. Cell. Biol. 144, 673-685 (1999).

33. Ito, J., Nagayasu, Y., Ueno, S. \& Yokoyama, S. Apolipoprotein-mediated cellular lipid release requires replenishment of sphingomyelin in a phosphatidylcholine-specific phospholipase C-dependent manner. J. Biol. Chem. 277, 44709-44714 (2002).

34. Yasuda, S. et al. A novel inhibitor of ceramide trafficking from the endoplasmic reticulum to the site of sphingomyelin synthesis. J. Biol. Chem. 276, 43994-44002 (2001).

35. Fukunaga, T. et al. Implication of sphingolipid metabolism in the stability of the Golgi apparatus. J. Cell. Sci. 113(Pt 18), 3299-3307 (2000).

36. Kim, Y. C. \& Guan, K. L. mTOR: a pharmacologic target for autophagy regulation. J. Clin. Invest. 125, 25-32 (2015).

37. Sarkar, S. Regulation of autophagy by mTOR-dependent and mTORindependent pathways: autophagy dysfunction in neurodegenerative diseases and therapeutic application of autophagy enhancers. Biochem. Soc. Trans. 41, 1103-1130 (2013).

38. Kim, J., Kundu, M., Viollet, B. \& Guan, K. L. AMPK and mTOR regulate autophagy through direct phosphorylation of Ulk1. Nat. Cell Biol. 13, 132-141 (2011).

39. Moruno-Manchon, J. F., Perez-Jimenez, E. \& Knecht, E. Glucose induces autophagy under starvation conditions by a p38 MAPK-dependent pathway. Biochem. J. 449, 497-506 (2013).

40. Heras-Sandoval, D., Perez-Rojas, J. M., Hernandez-Damian, J. \& Pedraza-Chaverri, J. The role of PI3K/AKT/mTOR pathway in the modulation of autophagy and the clearance of protein aggregates in neurodegeneration. Cell. Signal. 26, 2694-2701 (2014).
41. Galadari, S., Rahman, A., Pallichankandy, S., Galadari, A. \& Thayyullathil, F. Role of ceramide in diabetes mellitus: evidence and mechanisms. Lipids Health Dis. 12 98 (2013).

42. Chen, M. B. et al. C6 ceramide dramatically increases vincristine sensitivity both in vivo and in vitro, involving AMP-activated protein kinase-p53 signaling. Carcinogenesis 36, 1061-1070 (2015).

43. Liangpunsakul, S. et al. Inhibitory effect of ethanol on AMPK phosphorylation is mediated in part through elevated ceramide levels. Am. J. Physiol. Gastrointest. Liver Physiol. 298, G1004-G1012 (2010).

44. Wu, Y., Song, P., Xu, J., Zhang, M. \& Zou, M. H. Activation of protein phosphatase $2 \mathrm{~A}$ by palmitate inhibits AMP-activated protein kinase. J. Biol. Chem. 282, 9777-9788 (2007).

45. Pickrell, A. M. \& Youle, R. J. The roles of PINK1, parkin, and mitochondrial fidelity in Parkinson's disease. Neuron 85, 257-273 (2015).

46. Munoz, P., Huenchuguala, S., Paris, I. \& Segura-Aguilar, J. Dopamine oxidation and autophagy. Park. Dis. 2012, 920953 (2012).

47. Guenther, G. G. et al. Ceramide starves cells to death by downregulating nutrient transporter proteins. Proc. . Natl. Acad. Sci. USA 105, 17402-17407 (2008).

48. Ordonez, R. et al. Ceramide metabolism regulates autophagy and apoptotic cell death induced by melatonin in liver cancer cells. J. Pineal Res. 59, 178-189 (2015).

49. Sentelle, R. D. et al. Ceramide targets autophagosomes to mitochondria and induces lethal mitophagy. Nat. Chem. Biol. 8, 831-838 (2012).

50. Yu, L. Recent progress in autophagy. Cell Res. 24, 1-2 (2014).

51. He, H. et al. Calcineurin suppresses AMPK-dependent cytoprotective autophagy in cardiomyocytes under oxidative stress. Cell Death Dis. 5, e997 (2014).

52. Funato, K. \& Riezman, H. Vesicular and nonvesicular transport of ceramide from ER to the Golgi apparatus in yeast. J. Cell. Biol. 155, 949-959 (2001).

53. Gu, L. et al. Early activation of nSMase2/ceramide pathway in astrocytes is involved in ischemia-associated neuronal damage via inflammation in rat hippocampi. J. Neuroinflamm. 10, 109 (2013).

54. Clarke, C. J., Truong, T. G. \& Hannun, Y. A. Role for neutral sphingomyelinase-2 in tumor necrosis factor alpha-stimulated expression of vascular cell adhesion molecule-1 (VCAM) and intercellular adhesion molecule-1 (ICAM) in lung epithelial cells: p38 MAPK is an upstream regulator of nSMase2. J. Biol. Chem. 282, 1384-1396 (2007).

55. Lopez-Montero, I., Velez, M. \& Devaux, P. F. Surface tension induced by sphingomyelin to ceramide conversion in lipid membranes. Biochim. Biophys. Acta 1768, 553-561 (2007).

56. Stoffel, W. et al. Neutral sphingomyelinase (SMPD3) deficiency disrupts the Golgi secretory pathway and causes growth inhibition. Cell Death Dis. 7, e2488 (2016).

57. Yen, W. L. et al. The conserved oligomeric Golgi complex is involved in double-membrane vesicle formation during autophagy. J. Cell. Biol. 188 , 101-114 (2010).

58. Geng, J. \& Klionsky, D. J. The Golgi as a potential membrane source for autophagy. Autophagy 6, 950-951 (2010).

59. Komatsu, M. et al. Loss of autophagy in the central nervous system causes neurodegeneration in mice. Nature 441, 880-884 (2006).

60. Dauer, W. \& Przedborski, S. Parkinson's disease: mechanisms and models. Neuron 39, 889-909 (2003).

61. Reeve, A., Simcox, E. \& Turnbull, D. Ageing and Parkinson's disease: why is advancing age the biggest risk factor? Ageing Res. Rev. 14, 19-30 (2014).

62. Hebbar, S. et al. Ceramides And Stress Signalling Intersect With Autophagic Defects In Neurodegenerative Drosophila blue cheese (bchs) Mutants. Sci. Rep. 5, 15926 (2015)

63. He, Q. et al. Primary cilia in stem cells and neural progenitors are regulated by neutral sphingomyelinase 2 and ceramide. Mol. Biol. Cell. 25, 1715-1729 (2014).

64. Dinkins, M. B. et al. Neutral Sphingomyelinase-2 Deficiency Ameliorates Alzheimer's Disease Pathology and Improves Cognition in the 5XFAD Mouse. J. Neurosci.: Off. J. Soc. Neurosci. 36, 8653-8667 (2016).

65. Chu, T. H. et al. Axonal and myelinic pathology in 5xFAD Alzheimer's mouse spinal cord. PLoS One 12, e0188218 (2017).

66. Chong, F. P., Ng, K. Y., Koh, R. Y. \& Chye, S. M. Tau proteins and tauopathies in Alzheimer's disease. Cell. Mol. Neurobiol. 38, 965-980 (2018).

67. Schaeffer, $V$. et al. Stimulation of autophagy reduces neurodegeneration in a mouse model of human tauopathy. Brain: a J. Neurol. 135, 2169-2177 (2012). 
68. Caccamo, A., Majumder, S., Richardson, A., Strong, R. \& Oddo, S. Molecular interplay between mammalian target of rapamycin (mTOR), amyloid-beta, and Tau: effects on cognitive impairments. J. Biol. Chem. 285, 13107-13120 (2010).

69. Avrahami, L. et al. Inhibition of glycogen synthase kinase-3 ameliorates betaamyloid pathology and restores lysosomal acidification and mammalian target of rapamycin activity in the Alzheimer disease mouse model: in vivo and in vitro studies. J. Biol. Chem. 288, 1295-1306 (2013).
70. Tabatadze, N. et al. Inhibition of neutral sphingomyelinase-2 perturbs brain sphingolipid balance and spatial memory in mice. J. Neurosci. Res. 88 2940-2951 (2010).

71. Chu, C. T. et al. Autophagy in neurite injury and neurodegeneration: in vitro and in vivo models. Methods Enzymol. 453, 217-249 (2009).

72. Chiu, K., Lau, W. M., Lau, H. T., So, K. F., Chang, R. C. Micro-dissection of rat brain for RNA or protein extraction from specific brain region. J. Vis. Exp.: JoVE 7, e269 (2007) 